\title{
NON-HOMOGENEOUS DISSOLUTION OF DRUG AFFECTS THE QUALITY OF PARENTERAL TREATMENT
}

\author{
PAVLÍNA ŠTRBOVÁ, PETRA MATALOVÁ, KAREL URBÁNEK \\ and MARTIN PORUBA \\ Department of Pharmacology, Faculty of Medicine and Dentistry, \\ Palacký University in Olomouc, Olomouc, Czech Republic
}

\begin{abstract}
Intravenous administration of the drugs poses risks as treatment failure or higher incidence of unwanted effects. There are many critical steps in the preparation of infusion. The aim of this experimental study was to evaluate the effect of careful or careless infusion preparation on meropenem concentration in the infusion. Three different ways for the preparation of the infusion solution were compared. The first third of the bags were inverted ten times after meropenem addition. The second third of the bags were hung up after meropenem addition without shaking, and the last third of the bags were first hang up on the holder and then the antibiotic was added. Samples of meropenem were collected regularly and were used for the subsequent HPLC analysis. There are significant differences in meropenem concentration depending on the preparation. The variation of meropenem concentration is significantly higher where inadequate homogenization was used. Our results showed that proper inverting of the infusion container provides the homogenous infusion solution. Late drug addition or inadequate mixing may have a clinical impact on the given dose, especially in continuously administered drugs.
\end{abstract}

Keywords: infusion therapy, meropenem, drug dissolution, drug homogenization, drug concentration

Administration of drugs by intravenous infusion is a mainstay in many fields of medicine (1). Intravenous medications pose particular risks because of their greater complexity and the multiple steps required in their preparation, administration, and monitoring. These processes present numerous opportunities for errors. According to a prospective observational study, the rate of serious clinical errors is $25.5 \%$ (2). Some of these errors may affect drug concentration in an infusion vehicle. An incorrect concentration of a drug may influence treatment efficacy or the incidence of unwanted effects of the treatment. There are studies that support our hypothesis that inadequate mixing of medication in infusion vehicle may result in the layering of a drug in the vehicle $(3,4)$. We expect - and some studies support our expectations - that the drug may accumulate at the bottom of the container and the patient will receive a highly concentrated solution at the start of the therapy. On the other hand, the drug concentration will decrease depending on the duration of infusion therapy and, at the end of the therapy, the patient may suffer from underdosing.
As a model drug, the antibiotic meropenem was chosen, because beta-lactam antibiotics are among the most widely prescribed antibiotics in the ICU and have time-dependent bactericidal activity (5). According to the manufacturer, meropenem infusion should not exceed 30 minutes (6); some recent studies, however, suggest that prolonged infusion of meropenem is more effective than the conventional duration of the infusion $(7,8)$. For this reason, a simulation of prolonged infusion was used for our experiment.

The aim of this study was to support the general recommendation to homogenize the infusion solution prior to use and prevent the use of infusion containers without proper shaking. More specifically, we compared the effect of vigorous homogenization of drug in the vehicle and careless preparation of infusion.

\section{EXPERIMENTAL}

The Orchestra Module MVP PT infusion pump (Fresenius Kabi); flexible PVC-free infusion bags

\footnotetext{
* Corresponding author: e-mail: martin.poruba@upol.cz
} 
containing $250 \mathrm{~mL}$ normal saline solution (Baxter Healthcare Limited, Thetford, UK; Lot. No.: 250 mL: 14I13E4L); GAMA-IS infusion set (Gama Group a.s., Jimramov, Czech Republic, Lot. No.: 140020); and Meropenem Kabi (Fresenius Kabi Deutschland GmbH, Bad Homburg, Deutschland; Lots. No.: 0002D0 and 0007D0) were used for the experiment. Analytical standard of meropenem (content $=98 \%$ ) for validation of HPLC method was obtained from Cayman chemical company (Michigan, USA). Potassium dihydrogen phosphate (p.a., content $=99 \%$ ) for the preparation of a mobile phase was obtained from Penta (Prague, Czech Republic). All remaining chemicals were purchased from VWR International (Randnor, Pennsylvania, USA).

All procedures were conducted under standard laboratory conditions simulating hospital conditions. A total of 30 infusion bags were used for the study. All containers had been spiked by the GAMA-IS infusion set before meropenem was added to each container. Meropenem in form of powder was reconstituted in $10 \mathrm{~mL}$ of saline and prepared solution was then used for the preparing of infusion solution. The first third of the bags (B1) were carefully shaken after the addition of the antibiotic. The mixing was carried out by turning the bags upside down ten times. These bags simulated a well-mixed and homogeneous solution of the drug in normal saline solution. The second third of the bags (B2) were hung up after meropenem addition without shaking. These bags simulated a careless preparation of a mixture of the drug in infusion vehicle. The last third of the bags (B3) were first hung up on the holder and then meropenem was added. These bags simulated a delayed addition of the drug to the ongoing infusion therapy. Total time of two hours and a constant flow rate of $125 \mathrm{~mL} / \mathrm{h}$ were set on the infusion pump. Samples were collected at times $0\left(\mathrm{~T}_{0}\right), 30\left(\mathrm{~T}_{1}\right), 60\left(\mathrm{~T}_{2}\right), 90\left(\mathrm{~T}_{3}\right)$, and $120\left(\mathrm{~T}_{4}\right)$ minutes from the start of the infusion. A total of 150 samples (30 samples of each time fraction) were collected and all samples were immediately used for subsequent HPLC analysis.

Samples were diluted in mobile phase consisting of $10 \mathrm{mM}$ phosphate buffer with $\mathrm{pH} 7$ (filtered through hydrophilic polypropylene membrane with the pore size $0.45 \mu \mathrm{m}$ ) and acetonitrile (for HPLC, super gradient grade, content $=99.9 \%$ ) in a ratio $17: 3(\mathrm{v} / \mathrm{v})$. Following that, the samples were injected onto the HPLC system (LC-20A prominence, Shimadzu, Kyoto, Japan). Samples were separated with the mobile phase with a constant flow rate of $0.8 \mathrm{~mL} / \mathrm{min}$ and the elution was isocratic. Detection of analyte was set to a wavelength of $300 \mathrm{~nm}$.

The mean concentration of meropenem and standard deviations (SD) were calculated with the use of a calibration curve. For a statistical analysis of mean antibiotic concentration differences, the Kruskal-Wallis test was chosen. Differences were considered to be statistically significant when $p<0.05$. The statistical analysis was performed using the Statistica Cz 12 software (StatSoft CR, Prague, Czech Republic).

\section{RESULTS}

The HPLC method for determination of meropenem in saline solution was validated according to an agreement with ICH validation guidelines. The calibration curve is linear between the concentration range from 1 to $200 \mu \mathrm{g} / \mathrm{mL}$ with the linear correlation coefficient $\left(\mathrm{R}^{2}\right) 0.9995$. Concentrations of meropenem in all measured samples were within the calibration curve. Detection limit of quantification as a signal to noise ratio $10: 1$ was for this method

Table 1. Precision and accuracy as validation parameters of HPLC method for determination of meropenem in saline solution.

\begin{tabular}{|c|c|c|}
\hline Concentrations of calibration curve $(\mu \mathrm{g} / \mathrm{mL})$ & Precision $(\%)$ & Accuracy $(\%)$ \\
\hline 1 & 3.05 & 103.55 \\
\hline 10 & 0.88 & 95.33 \\
\hline 20 & 2.54 & 99.78 \\
\hline 40 & 1.77 & 95.58 \\
\hline 60 & 0.92 & 99.63 \\
\hline 80 & 0.06 & 99.53 \\
\hline 100 & 0.76 & 99.94 \\
\hline 120 & 0.02 & 101.36 \\
\hline 200 & 0.46 & 102.14 \\
\hline
\end{tabular}




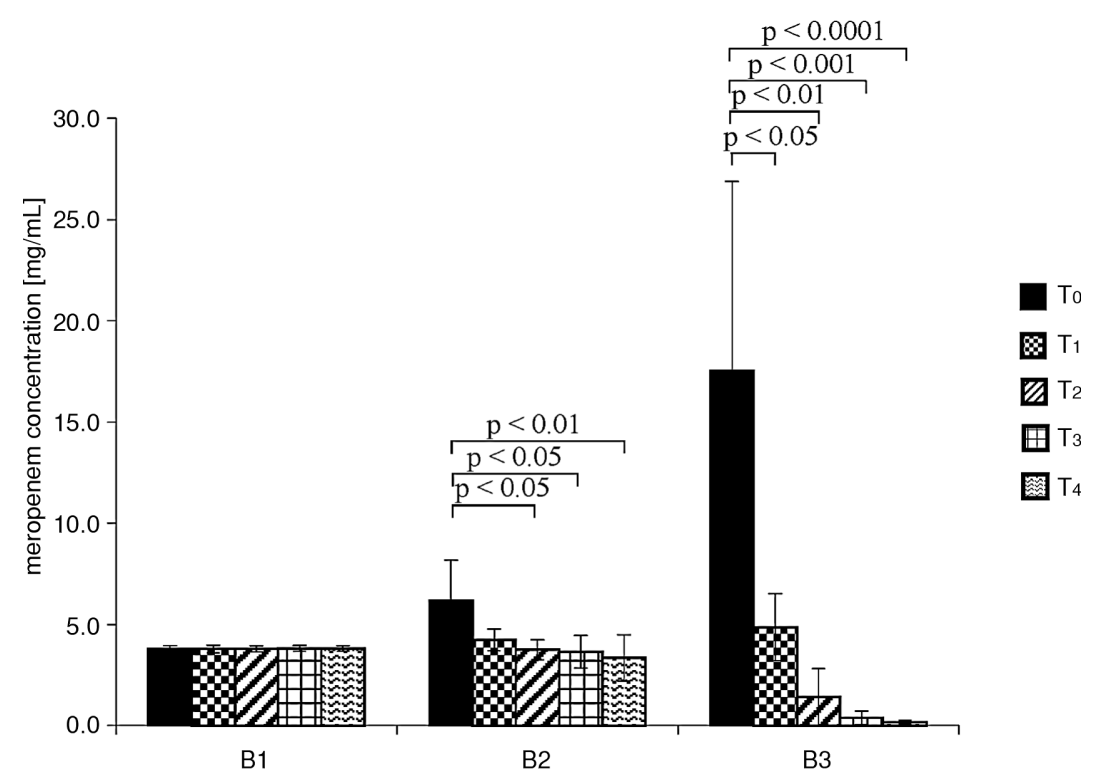

Figure 1. Differences in meropenem concentration within B1, B2, and B3. Data are expressed as means $\pm \mathrm{SD}, \mathrm{n}=10 .{ }^{*} p<0.05$, $* * p<0.01, * * * p<0.001, * * * * p<0.0001$

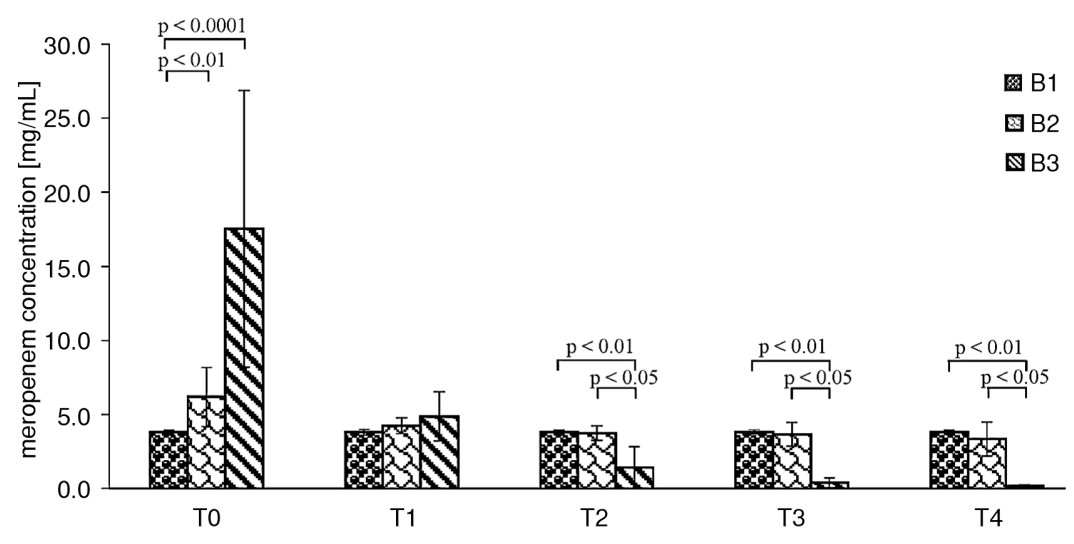

Figure 2. Differences in meropenem concentration across B1, B2, and B3. Data are expressed as means $\pm \mathrm{SD}, \mathrm{n}=10 .{ }^{*} p<0.05$, $* * p<0.01, * * * p<0.001, * * * * p<0.0001$

measured as $0.5 \mu \mathrm{g} / \mathrm{mL}$ (this value was also measured in agreement with ICH validation). Values of precision and accuracy are shown in Table 1. Only meropenem was detected in analyses, no impurities were present in samples.

Figure 1 shows the measured concentrations of meropenem in all the samples collected. There were no differences in drug concentration within the B1 samples. Figure 1 also shows the statistically signif- icant differences in meropenem concentrations within the B2 and B3 samples. For the B2 samples, the mean drug concentration of the samples collected at time $0\left(\mathrm{~T}_{0}\right)$ was $6.2 \mathrm{mg} / \mathrm{mL}$. The samples collected at $\mathrm{T}_{1}$ showed a 1.5-fold decrease compared with $\mathrm{T}_{0}$ without statistical significance. Samples $\mathrm{T}_{2}, \mathrm{~T}_{3}$, and $\mathrm{T}_{4}$ showed a 1.7 -fold $(p<0.05)$, a 1.7 -fold $(p<$ $0.05)$, and a 1.8 -fold $(p<0.01)$ decrease compared with $\mathrm{T}_{0}$ with statistical significance. For the B3 sam- 
ples, the mean meropenem concentration in $\mathrm{T}_{0}$ samples was $17.4 \mathrm{mg} / \mathrm{mL}$. Our results showed a 3.6-fold $(p<0.05)$, a 12.3-fold $(p<0.01)$, a 45.2-fold $(p<$ $0.001)$, and 104.6-fold $(p<0.0001)$ decrease in drug concentration in the $\mathrm{T}_{1}, \mathrm{~T}_{2}, \mathrm{~T}_{3}$, and $\mathrm{T}_{4}$ samples, respectively, compared with $\mathrm{T}_{0}$.

As shown in Figure 2, the measured drug concentration of the B1 samples was $3.8 \mathrm{mg} / \mathrm{mL}$. The meropenem concentration at $\mathrm{T}_{0}$ was 1.6-fold $(p<$ $0.01)$ and 4.6-fold $(p<0.0001)$ increased in the $\mathrm{B} 2$ and B3 samples, respectively, compared with B1. At the same time, the concentration was 2.8-fold higher in B3 compared with B2. This increase in drug concentration failed to reach statistical significance. At the $\mathrm{T}_{1}$ time, no significant differences in drug concentrations across all the samples were observed. At the $\mathrm{T}_{2}, \mathrm{~T}_{3}$, and $\mathrm{T}_{4}$ times, the measured meropenem concentrations in B3 were 2.7-fold $(p<0.01)$, 9.8-fold $(p<0.01)$, and 22.7-fold $(p<0.01)$ decreased in comparison with B1; and 2.6-fold ( $p<$ $0.05)$, 9.4-fold $(p<0.05)$, and 20.0-fold $(p<0.05)$ decreased compared with B2. At the latter times, there were no statistically significant differences between $\mathrm{B} 1$ and the $\mathrm{B} 2$.

\section{DISCUSSION AND CONCLUSION}

We hypothesized that inadequate dissolution and homogenization of a drug in infusion vehicle may contribute to treatment failure and a higher incidence of unwanted effects. The parenteral administration of a drug in normal saline was simulated and the time-dependent concentrations of the drug in infusion vehicle were measured.

Our results show that careless preparation of infusion without proper shaking may result in a significant variation in drug concentration during infusion. Inadequate mixing or shaking of admixed meropenem leads to layering of the drug in the solution. Based on our results, the concentration variability of meropenem highly depends on the method of infusion preparation. Layering of the drug in infusion fluid was most pronounced in the bags in which meropenem was added following container hang-up. In the B2 bags, moving the containers slightly after the addition of the drug probably caused mild mixing of this mixture. For this reason, a lower variability of meropenem concentration was seen in these infusion containers.

Donaldson et al. showed that there were important differences in electrolyte concentration depending on the device used and time delay before infusion. Electrolyte concentrations are less variable when are prepared in PVC bags rather than syringes or when the solution is vigorously mixed or allowed to stand for a period of 24 hours (9). In our study, we started to simulate infusion as early as possible according to the instruction that the solution prepared should be used as soon as practicable after reconstitution (6). The negative consequences are related to the drug used. Meropenem is an antibiotic with the time-dependent killing of bacteria. Higher concentration of an antibiotic at the start and low concentration at the end of the infusion may shorten the time of the antibiotic plasma concentration exceeding its minimal inhibitory concentration (MIC) to bacterial pathogen. Our results are relatable to any other drug that can be mixed with infusion vehicle in an infusion container, particularly to those administered by continuous infusion and with a low therapeutic index.

For the preparation of an effective and safe infusion solution, proper mixing is necessary. Based on our results, turning the bags upside down ten times is sufficient. This process provides the required homogenization of solution without unwanted layering of the drug on the bottom of the infusion container. On the other hand, a careless preparation of infusion solution without any shaking or mixing should be avoided in the clinical practice. Previously published and cited studies did not compare the drug concentration following the addition of a drug to the ongoing infusion. We suggest the addition of the drug to the container during infusion should be restricted only to emergent cases and drugs with a high therapeutic index. Nurses should be educated to not underestimate precise mixing and complete homogenization of infusion solutions.

\section{Acknowledgments}

This study was supported by grant IGA_LF_2018_011.

\section{Conflicts of interest}

Authors declare no conflict of interest.

\section{REFERENCES}

1. Adapa R.M., Mani V., Murray L.J., Degnan B.A., Ercole A. et al.: Br. J. Anaesth. 109, 729 (2012).

2. Westbrook J.I., Rob M.I., Woods A., Parry D.: BMJ Qual. Saf. 20, 1027 (2011).

3. Thompson W.L., Feer T.D.: Crit. Care Med. 8, 603 (1980).

4. Bergman N., Vellar I.D.: Med. J. Aust. 6, 270 (1982). 
5. Ulldemolins M., Vaquer S., Llaurado-Serra M., Pontes C., Calvo G. et al.: Crit. Care. 18, 227 (2014).

6. https://www.fresenius-kabi.com/au/documents/ Meropenem_PI.pdf. (accessed on 11. 07. 2017).

7. Vourli S., Tsala M., Kotsakis S., Daikos G.L., Tzouvelekis L. et al.: J. Pharm. Sci. 105, 1513 (2016).
8. Shabaan A.E., Nour I., Elsayed Eldegla H., Nasef N., Shouman B. et al.: Pediatr. Infect. Dis. J. 36, 358 (2017).

9. Donaldson T.M., Mani V., Wheeler D.W.: Postgrad. Med. J. 87, 83 (2011).

Received: 6.08. 2018 